\title{
Hibiscus Sabdariffa L. UKMR-2 AS A Protective Agent Against Sperm and Testicular Damage IN Streptozotocin Induced Diabetic Rats
}

\author{
Muhd Hanis Md Idris ${ }^{1, *}$, Siti Balkis Budin ${ }^{1}$, Mohamad Osman $^{2}$ and Jamaludin Mohamed ${ }^{1}$

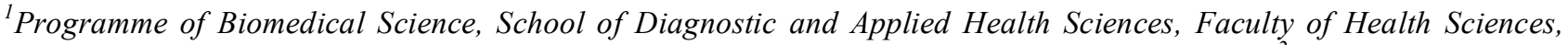 \\ Universiti Kebangsaan Malaysia, Jalan Raja Muda Abdul Aziz, 50300 Kuala Lumpur, Malaysia, ${ }^{2}$ Faculty of Plantation \\ and Agrotechnology, Universiti Teknologi MARA, 40450 Shah Alam, Selangor, Malaysia; \\ E-mail:muhd_hanis@hotmail.com
}

Diabetes mellitus play a very important role in male sexual dysfunction and infertility as consequences of oxidative damage. Hibiscus sabdariffa exerts natural antioxidant properties as shown in experimental studies. This study was designed to investigate the role of $H$. sabdariffa UKMR-2 (HSE) on sperm characteristics and hormonal changes in streptozotocin induced diabetic rats. Male Sprague-Dawley rats were allotted into four groups: control, HSE, diabetes and diabetes+HSE. HSE (100 $\mathrm{mg} / \mathrm{kg}$ body weight) was administered orally for 28 consecutive days. At the end of experiment, plasma was collected and cauda epididymis was dissected out to obtain sperm. In diabetic+HSE group, sperm motility, viability and concentration were significantly higher $(p<0.05)$ than diabetic group. Contrarily, sperm abnormal morphology was significantly lower $(p<0.05)$ in diabetic + HSE group as compared to diabetic group. Follicle-stimulating hormone (FSH) level was significantly higher $(p<0.05)$ in diabetic + HSE group than diabetic group. Testosterone level was slightly higher in diabetic+HSE group than diabetic group while luteinizing hormone (LH) level was lowered in diabetic+HSE group than diabetic group. However, no significant differences were seen in both parameters. Histological observation revealed that degenerative changes such as disorganization and degeneration of seminiferous tubules and defection in Sertoli cells and spermatogonia occurred in testis of diabetic rats. Otherwise, HSE supplementation ameliorated this alteration. On the other hand, phytochemical study of $H$. sabdariffa UKMR2 showed that it possessed the property of alkaloid, flavanoid, saponin, tannins, cardiac glycoside and anthraquinones. In conclusion, the present study suggests that HSE has a potential in preventing sperm and testicular alteration in diabetic rat models.

Keywords: Hibiscus sabdariffa, UKMR-2, Sperm, Testis, Diabetes. 